\title{
The Dispersion Relation of Internal Wave Extended-Korteweg-de Vries Equation in a Two-Layer Fluid
}

\author{
Pingping Feng, Xianghua Meng* \\ School of Applied Science, Beijing Information Science and Technology University, Beijing, China \\ Email: *xhmeng@bistu.edu.cn
}

How to cite this paper: Feng, P.P. and Meng, X.H. (2021) The Dispersion Relation of Internal Wave Extended-Korteweg-de Vries Equation in a Two-Layer Fluid. Journal of Applied Mathematics and Physics, 9 , 1056-1064.

https://doi.org/10.4236/jamp.2021.95072

Received: December 16, 2020

Accepted: May 24, 2021

Published: May 27, 2021

Copyright $\odot 2021$ by author(s) and Scientific Research Publishing Inc. This work is licensed under the Creative Commons Attribution International License (CC BY 4.0).

http://creativecommons.org/licenses/by/4.0/

\section{(c) (i) Open Access}

\begin{abstract}
To understand the characteristics of ocean internal waves better, we study the dispersion relation of extended-Korteweg-de Vries (EKdV) equation with quadratic and cubic nonlinear terms in a two-layer fluid by using the Poincaré-Lighthill-Kuo (PLK) method which is one of the perturbation methods. Starting from the partial differential equation, the PLK method can be used to solve the dispersion relation of the equation. In this paper, we use PLK method to solve the equation and derive the dispersion relation of EKdV equation which is related to wave number and amplitude. Based on the dispersion relation obtained in this paper, the expressions of group velocity and phase velocity of the equation are obtained. Under the actual hydrological data, the influence of hydrological parameters on the dispersion relation for descending internal wave is discussed. It is hope that the obtained results will be helpful to the study of energy transfer and other internal wave parameters in the future.
\end{abstract}

\section{Keywords}

Ocean Internal Waves, Dispersion Relation, Extended-Korteweg-de Vries Equation, Poincaré-Lighthill-Kuo Method

\section{Introduction}

Ocean internal solitary wave is a kind of internal waves, which is the result of dispersion effect and nonlinear effect [1]. Under the balance of nonlinear effect and dispersion effect, the waveform can keep constant for hundreds of kilometers in the process of propagation [2] [3]. The dispersion relation is the basis of studying ocean internal solitary waves [4]. The dispersion relation describes the relationship between wave frequency and wave number. The expressions of the 
phase velocity and the group velocity obtained from dispersion relation which can reflect the propagation of internal wave signal and energy can be calculated respectively [5]. The energy exchange in the ocean caused by the occurrence and evolution of internal solitary waves provides abundant nutrients and living space for marine organisms, especially ephemeroptera plants [6] and the shear flow caused by energy exchange seriously threatens the marine operations and military activities. Therefore, the investigation of dispersion relation of internal waves is of great significance to scientific research, marine engineering security, national defense security and marine biological transportation [7]. Hence, this is the main reason why the theoretical dispersion relation of the internal solitary waves equation is widely studied [8]-[13].

Starting from modal equation, Fliegel and Haskell used Thomson-Haskell method to calculate the dispersion relation of internal waves [8]; Munk derived the wave function in the form of Airy function and the corresponding dispersion relation in integral form when the frequency of internal wave was close to Brunt-Väisälä frequency N [14]; Wang and Shang used WKB method to study the dispersion relation of internal wave when the floating frequency was a slow varying function of water depth and internal wave frequency was close to Brunt-Väisälä frequency N [15]; Zhang and Gao solved the vertical structure and dispersion relation of internal waves by using the transformation method of Russian scholars [16]. In reference [17], the dispersion relation of internal solitary waves of the $\mathrm{KdV}$ equation was obtained from nonlinear partial differential equations.

PLK method was first proposed by Poincaré in finding the periodic solutions of the first-order ordinary differential equations. Later, Lighthill made an important promotion in finding the uniformly effective approximate solution of physical problems. Finally, Kuo further extended Lighthill's original idea in seeking the elegant solution of the incompressible laminar boundary layer of a flat plate and subsequent work [18]. For the conventional perturbation method, only the dependent variable in the original function is expanded by perturbation, while the PLK method also expands the circular frequency by perturbation. Even if the equation is not integrable, the PLK method can be used to calculate the dispersion relation of the equation.

In this paper, we study the extended-Korteweg-de Vries (EKdV) equation with quadratic and cubic nonlinear terms proposed by T. Sakai and L. G. Redekopp which can better describe large amplitude waves propagation problem [19]. The dispersion relation with the perturbation solution of EKdV equation is obtained by using PLK method. Based on the dispersion relation, the expressions of group velocity and phase velocity are obtained. The effect of wave depth and density difference ratio on the dispersion relation of the EKdV equation is discussed for descending ocean internal waves.

\section{The Dispersion Relation of the EKdV Equation}

Grimshaw first describes the weakly nonlinear evolution of interfacial gravity 
waves on two shallow boundaries with $\mathrm{KdV}$ equation [20] [21]. When an extended $\mathrm{KdV}$ equation is used, the agreement between the theoretical and experimental data is greatly improved [20] [21] [22]. In view of this, T. Sakai and L. G. redekopp proposed the EKdV equation which can describe the internal wave packet with large amplitude. The two-layer EKdV equation form is as follows

$$
\zeta_{t}+c_{0}\left(1-\alpha_{1} \zeta-\alpha_{2} \zeta^{2}\right) \zeta_{x}+\beta_{0} c_{0} \zeta_{x x x}=0
$$

The coefficients of Equation (1) are as follows:

$$
\begin{gathered}
c_{0}^{2}=\frac{\tilde{g} h_{1} h_{2}}{h_{1}+h_{2}}, \\
\alpha_{1}=\frac{3}{2} \frac{h_{2}-h_{1}}{h_{1} h_{2}}, \\
\alpha_{2}=\frac{3}{8} \frac{\left(h_{2}-h_{1}\right)^{2}+8 h_{1} h_{2}}{\left(h_{1} h_{2}\right)^{2}}, \\
\beta_{0}=\frac{1}{6} h_{1} h_{2},
\end{gathered}
$$

where $c_{0}$ is the linear velocity, $\alpha_{1}$ is the quadratic nonlinear term, $\alpha_{2}$ is the cubic nonlinear term, $\beta_{0}$ is the dispersion coefficient and gravity

$\tilde{g}=g \frac{\rho_{2}-\rho_{1}}{\rho_{1}}=g \frac{\Delta \rho}{\rho_{1}}, \rho_{1}$ and $\rho_{2}$ are the densities of the upper and lower layers of sea water.

When $\alpha_{2} \neq 0$, Equation (1) is called KdV2 equation. When $\alpha_{2}=0$, Equation (1) is called KdV1 equation. KdV1 equation and KdV2 equation are both $\mathrm{KdV}$ families, but they are different [18]. The nonlinearity of KdV1 equation is completely derived from the leading nonlinear correction of the linear long wave phase velocity $c_{0}$, the long wave phase velocity of KdV2 equation is dependent on the first and second order terms of wave amplitude [19].

The dispersion relation of Equation (1) will be derived. Introducing dimensionless variation $\frac{h_{2}-h_{1}}{h_{1} h_{2}} \zeta=A$, Equation (1) can be transformed into

$$
A_{t}+c_{0} A_{x}-\frac{3}{2} c_{0} A A_{x}-c_{0} \frac{9 \alpha_{2}}{4 \alpha_{1}^{2}} A^{2} A_{x}+\beta_{0} c_{0} A_{x x x}=0 .
$$

Introducing phase function

$$
\xi=k x-\omega t
$$

where $k$ and $\omega$ are wave number and circular frequency respectively, Equation (6) becomes the following nonlinear ordinary differential equation

$$
-\omega \frac{\mathrm{d} A}{\mathrm{~d} \xi}+c_{0} k \frac{\mathrm{d} A}{\mathrm{~d} \xi}-\frac{3}{2} c_{0} k A \frac{\mathrm{d} A}{\mathrm{~d} \xi}-\frac{9}{4} c_{0} k \frac{\alpha_{2}}{\alpha_{1}^{2}} A^{2} \frac{\mathrm{d} A}{\mathrm{~d} \xi}+c_{0} \beta_{0} k^{3} \frac{\mathrm{d}^{3} A}{\mathrm{~d} \xi^{3}}=0 .
$$

Using the PLK method for Equation (7), the dispersion relation of Equation (1) is obtained. Let $\eta_{0}$ be the amplitude of $\zeta$, and select $\varepsilon$ as a small parameter 


$$
\varepsilon=\frac{h_{2}-h_{1}}{h_{1} h_{2}} \eta_{0}<1 .
$$

Both $A$ and $\omega$ are expanded to power series of $\varepsilon$. Because the dimensionless numbers $A$ and $\varepsilon$ are small quantities of the same order, $A$ is expanded from the first order of $\varepsilon$, and $\omega$ is expanded from the zero order. The perturbation expansion of $A$ with respect to $\varepsilon$ is written as

$$
A=\varepsilon A_{1}(\zeta)+\varepsilon^{2} A_{2}(\zeta)+\varepsilon^{3} A_{3}(\zeta)+\cdots,
$$

and the perturbation expansion of the circular frequency $\omega$ is written as

$$
\omega=\omega_{0}(k)+\varepsilon \omega_{1}(k)+\varepsilon^{2} \omega_{2}(k)+\cdots .
$$

Substituting Equation (9) and Equation (10) into Equation (7), we can get

$$
\begin{aligned}
& -\left(\omega_{0}+\varepsilon \omega_{1}+\varepsilon^{2} \omega_{2}\right) \frac{\mathrm{d}\left(\varepsilon A_{1}+\varepsilon^{2} A_{2}+\varepsilon^{3} A_{3}\right)}{\mathrm{d} \xi}+c_{0} k \frac{\mathrm{d}\left(\varepsilon A_{1}+\varepsilon^{2} A_{2}+\varepsilon^{3} A_{3}\right)}{\mathrm{d} \xi} \\
& -\frac{3}{2} c_{0} k\left(\varepsilon A_{1}+\varepsilon^{2} A_{2}+\varepsilon^{3} A_{3}\right) \frac{\mathrm{d}\left(\varepsilon A_{1}+\varepsilon^{2} A_{2}+\varepsilon^{3} A_{3}\right)}{\mathrm{d} \xi} \\
& +c_{0} \beta_{0} k^{3} \frac{\mathrm{d}^{3}\left(\varepsilon A_{1}+\varepsilon^{2} A_{2}+\varepsilon^{3} A_{3}\right)}{\mathrm{d} \xi^{3}} \\
& -\frac{9}{4} c_{0} k \frac{\alpha_{2}}{\alpha_{1}^{2}}\left(\varepsilon A_{1}+\varepsilon^{2} A_{2}+\varepsilon^{3} A_{3}\right)^{2} \frac{\mathrm{d}\left(\varepsilon A_{1}+\varepsilon^{2} A_{2}+\varepsilon^{3} A_{3}\right)}{\mathrm{d} \xi}=0 .
\end{aligned}
$$

The first-order to the third-order approximation is respectively

$$
\begin{gathered}
\left(k c_{0}-\omega_{0}\right) \frac{\mathrm{d} A_{1}}{\mathrm{~d} \xi}+c_{0} \beta_{0} k^{3} \frac{\mathrm{d}^{3} A_{1}}{\mathrm{~d} \xi^{3}}=0, \\
-\omega_{0} \frac{\mathrm{d} A_{2}}{\mathrm{~d} \xi}-\omega_{1} \frac{\mathrm{d} A_{1}}{\mathrm{~d} \xi}+c_{0} k \frac{\mathrm{d} A_{2}}{\mathrm{~d} \xi}-\frac{3}{2} c_{0} k A_{1} \frac{\mathrm{d} A_{1}}{\mathrm{~d} \xi}+c_{0} \beta_{0} k^{3} \frac{\mathrm{d}^{3} A_{2}}{\mathrm{~d} \xi^{3}}=0, \\
-\omega_{0} \frac{\mathrm{d} A_{3}}{\mathrm{~d} \xi}-\omega_{1} \frac{\mathrm{d} A_{2}}{\mathrm{~d} \xi}-\omega_{2} \frac{\mathrm{d} A_{1}}{\mathrm{~d} \xi}+c_{0} k \frac{\mathrm{d} A_{3}}{\mathrm{~d} \xi}-\frac{3}{2} c_{0} k A_{1} \frac{\mathrm{d} A_{2}}{\mathrm{~d} \xi}-\frac{3}{2} c_{0} k A_{2} \frac{\mathrm{d} A_{1}}{\mathrm{~d} \xi} \\
-\frac{9}{4} c_{0} k \frac{\alpha_{2}}{\alpha_{1}^{2}} A_{1}^{2} \frac{\mathrm{d} A_{1}}{\mathrm{~d} \xi}+c_{0} \beta_{0} k^{3} \frac{\mathrm{d}^{3} A_{3}}{\mathrm{~d} \xi^{3}}=0 .
\end{gathered}
$$

The first-order approximation Equation (12) is the second-order oscillation equation for $\frac{\mathrm{d} A_{1}}{\mathrm{~d} \xi} \cdot \varepsilon$ is dimensionless, hence

$$
\frac{c_{0} k-\omega_{0}}{c_{0} \beta_{0} k^{3}}=1 .
$$

The zero order approximation of circular frequency $\omega$ is

$$
\omega_{0}=c_{0} k-c_{0} \beta_{0} k^{3} .
$$

At the same time, the solution of the first-order approximation Equation (12) is obtained

$$
A_{1}=\sin \xi .
$$

Taking Equation (15) and Equation (16) into the second-order approximation 
Equation (13), we can get

$$
c_{0} \beta_{0} k^{3}\left(\frac{\mathrm{d} A_{2}}{\mathrm{~d} \xi}+\frac{\mathrm{d}^{3} A_{2}}{\mathrm{~d} \xi^{3}}\right)=\omega_{1} \cos \xi+\frac{3}{2} c_{0} k \sin \xi \cos \xi .
$$

It can be seen from the above formula that the non-duration condition here is

$$
\omega_{1}=0 .
$$

Then Equation (17) is reduced to

$$
\frac{\mathrm{d} A_{2}}{\mathrm{~d} \xi}+\frac{\mathrm{d}^{3} A_{2}}{\mathrm{~d} \xi^{3}}=\frac{3}{2 \beta_{0} k^{2}} \sin \xi \cos \xi .
$$

Its special solution is

$$
A_{2}=-\frac{1}{4 \beta_{0} k^{2}} \sin ^{2} \xi
$$

Taking Equations (15), (16), (18) and (20) into third-order approximation Equation (14), we can obtain

$$
c_{0} \beta_{0} k^{3}\left(\frac{\mathrm{d} A_{3}}{\mathrm{~d} \xi}+\frac{\mathrm{d}^{3} A_{3}}{\mathrm{~d} \xi^{3}}\right)=\omega_{2} \cos \xi+\left(-\frac{9}{8} \frac{c_{0}}{\beta_{0} k}+\frac{9}{4} c_{0} k \frac{\alpha_{2}}{\alpha_{1}^{2}}\right) \sin ^{2} \xi \cos \xi .
$$

It can be seen from the above formula that the non-duration condition here is

$$
\omega_{2}=-\frac{9\left(2 k^{2} \alpha_{2} \beta_{0}-\alpha_{1}^{2}\right) c_{0}}{32 k \beta_{0} \alpha_{1}^{2}} .
$$

And Equation (21) can be simplified as

$$
\frac{\mathrm{d} A_{3}}{\mathrm{~d} \xi}+\frac{\mathrm{d}^{3} A_{3}}{\mathrm{~d} \xi^{3}}=-\frac{9\left(2 k^{2} \alpha_{2} \beta_{0}-\alpha_{1}^{2}\right)}{32 \alpha_{1}^{2} \beta_{0}^{2} k^{4}} \cos \xi+\frac{9\left(2 \alpha_{2} \beta_{0} k^{2}-\alpha_{1}^{2}\right)}{8 \alpha_{1}^{2} \beta_{0}^{2} k^{4}} \sin ^{2} \xi \cos \xi .
$$

Its special solution is

$$
A_{3}=-\frac{3\left(2 k^{2} \alpha_{2} \beta_{0}-\alpha_{1}^{2}\right)}{64 \alpha_{1}^{2} \beta_{0}^{2} k^{4}} \sin ^{3} \xi .
$$

The perturbation solution of Equation (1) is obtained by using the PLK method

$$
A=\varepsilon \sin \xi-\varepsilon^{2} \frac{1}{4 \beta_{0} k^{2}} \sin ^{2} \xi-\varepsilon^{3} \frac{3\left(2 k^{2} \alpha_{2} \beta_{0}-\alpha_{1}^{2}\right)}{64 \alpha_{1}^{2} \beta_{0}^{2} k^{4}} \sin ^{3} \xi+o\left(\varepsilon^{4}\right) .
$$

The circular frequency is

$$
\omega=c_{0} k-c_{0} \beta_{0} k^{3}-\frac{9\left(2 k^{2} \alpha_{2} \beta_{0}-\alpha_{1}^{2}\right) c_{0}}{32 k \beta_{0} \alpha_{1}^{2}} \varepsilon^{2}+o\left(\varepsilon^{3}\right) .
$$

Noting that $\varepsilon=\frac{h_{2}-h_{1}}{h_{1} h_{2}} \eta_{0}$ and $\alpha_{1}=\frac{3}{2} \frac{h_{2}-h_{1}}{h_{1} h_{2}}$, the above formula can be adapted as

$$
\zeta=\eta_{0} \sin \xi-\frac{\alpha_{1}}{6 \beta_{0} k^{2}} \eta_{0}^{2} \sin ^{2} \xi-\frac{2 k^{2} \alpha_{2} \beta_{0}-\alpha_{1}^{2}}{48 \beta_{0}^{2} k^{4}} \eta_{0}^{3} \sin ^{3} \xi+o\left(\varepsilon^{4}\right) .
$$


And the circular frequency is

$$
\omega=c_{0} k-c_{0} \beta_{0} k^{3}-\frac{2 k^{2} \alpha_{2} \beta_{0}-\alpha_{1}^{2}}{8 k \beta_{0}} c_{0} \eta_{0}^{2}+o\left(\varepsilon^{3}\right) .
$$

Formula (27) is the dispersion relation of nonlinear internal solitary wave EKdV equation. By truncating formula (27), we can get the truncated expression of dispersion relation

$$
\omega=c_{0} k-c_{0} \beta_{0} k^{3}-\frac{2 k^{2} \alpha_{2} \beta_{0}-\alpha_{1}^{2}}{8 k \beta_{0}} c_{0} \eta_{0}^{2} .
$$

\section{Dispersion Relation Diagram Based on Actual Data}

Any wave equation has its specific dispersion relation, so we can determine the wave parameters of wave according to the dispersion relation. We select a set of data of Andaman Sea area to discuss the influence of hydrological parameters on dispersion relation. The water depth of upper layer $h_{1}=230 \mathrm{~m}$, the water depth of lower layer $h_{2}=863 \mathrm{~m}$, density difference ratio $\frac{\Delta \rho}{\rho}=0.003$, amplitude $\eta_{0}=60 \mathrm{~m} \quad$ [2]. For this set of data, the values of $\varepsilon$ is 0.191 , so that $|\varepsilon|<1$.

The expressions of phase velocity and group velocity can be calculated respectively based on the dispersion relation (28)

$$
\begin{gathered}
C_{p}=c_{0}-c_{0} \beta_{0} k^{2}-\frac{2 k^{2} \alpha_{2} \beta_{0}-\alpha_{1}^{2}}{8 k^{2} \beta_{0}} c_{0} \eta_{0}^{2}, \\
C_{g}=c_{0}-3 c_{0} \beta_{0} k^{2}-\frac{2 k^{2} \alpha_{2} \beta_{0}+\alpha_{1}^{2}}{8 k^{2} \beta_{0}} c_{0} \eta_{0}^{2} .
\end{gathered}
$$

Combining the expression of phase velocity [23]

$$
C_{p}=c_{0}\left(1+\frac{\eta_{0}\left(h_{2}-h_{1}\right)}{2 h_{1} h_{2}}\right),
$$

the value of wave number $k$ can be deduced by formula (29) and (31) using the measured data.

As seen from Figure 1, for the descending internal solitary wave, within a certain range of the water depth, when the lower layer water depth $h_{2}$ is fixed, the value of $\omega$ decreases with the increase of the upper layer water depth $h_{1}$ in Figure 1(a). When the upper layer water depth $h_{1}$ is fixed, the value of $\omega$ decreases with the increase of the lower layer water depth $h_{2}$ in Figure 1(b).

As seen from Figure 2, for the descending internal solitary wave, within a certain range of density difference ratio, the value of $\omega$ increases with the increase of density difference ratio.

\section{Conclusion}

In this paper, the dispersion relation with the perturbation solution of EKdV equation is obtained by using PLK method. The dispersion relation derived in this paper is related to water number and amplitude. The expressions of phase 


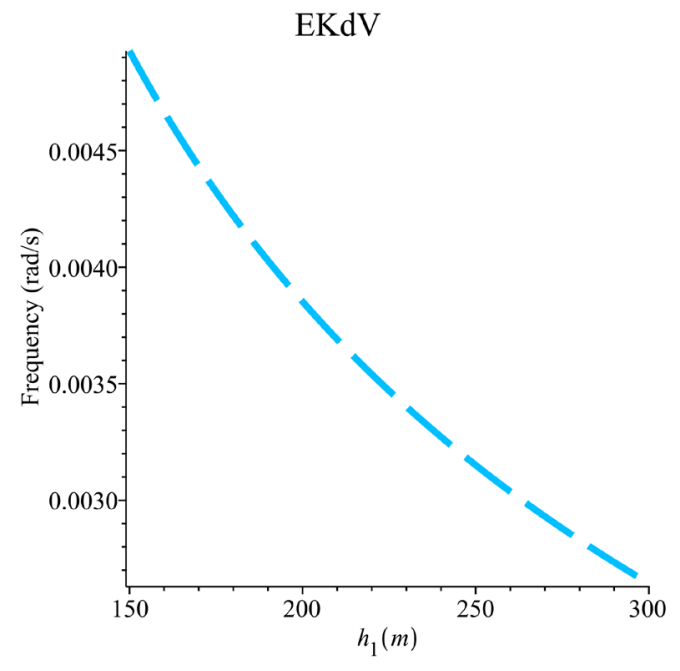

(a)

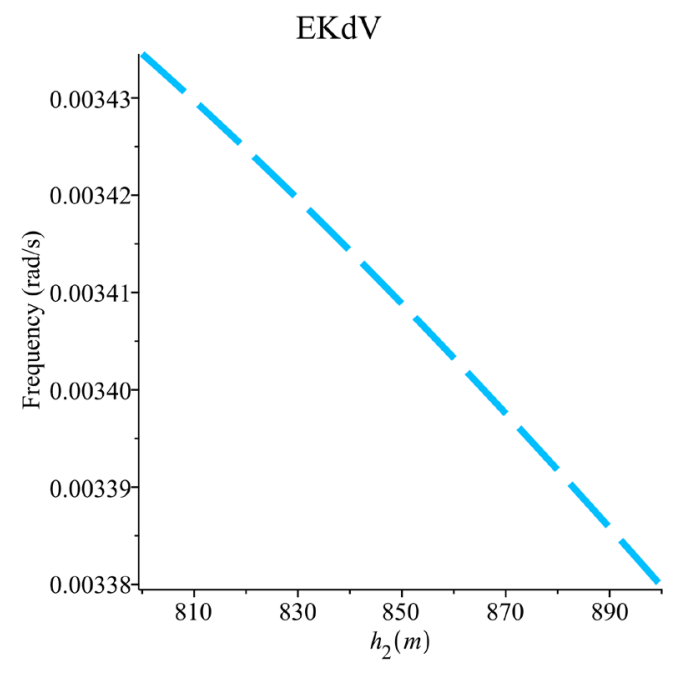

(b)

Figure 1. (a) The influence of the water depth of upper layer $h_{1}$ on $\omega$, (b) The influence of the water depth of lower layer $h_{2}$ on $\omega$.

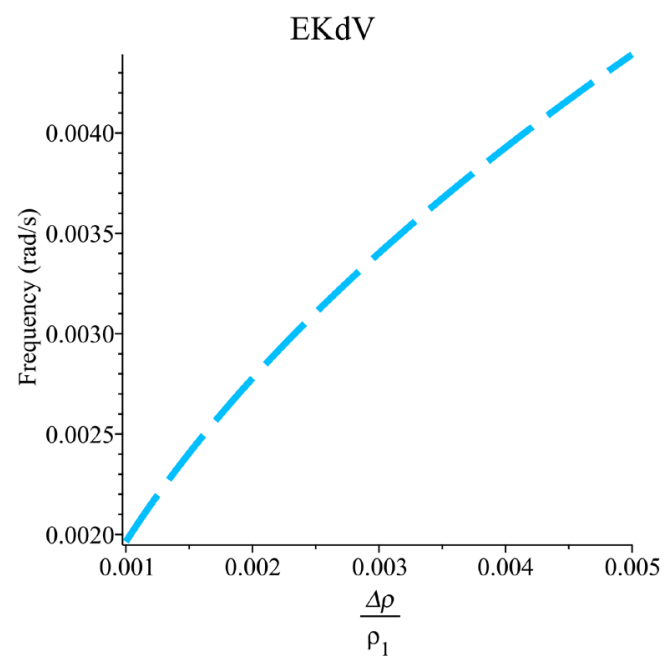

Figure 2. The influence of density difference ratio $\frac{\Delta \rho}{\rho_{1}}$ on $\omega$.

velocity and group velocity are obtained by using the dispersion relation, which can be used to study the propagation characteristics and energy transmission of ocean internal waves. Under the actual hydrological data, the influence of water depth and density difference ratio on the descending internal solitary waves is discussed. The value of $\omega$ decreases with the increase of the upper and lower water depth, but it increases with the increase of density difference ratio. We hope to provide a better theoretical basis for solving internal wave parameters by using dispersion relation.

\section{Acknowledgements}

This work has been supported by the project of Beijing Information Science and 
Technology University No. Z2018057.

\section{Conflicts of Interest}

The authors declare no conflicts of interest regarding the publication of this paper.

\section{References}

[1] Wang, Z.X. and Wang, C.X. (2019) Internal Solitary Wave and Interaction in the Northern South China Sea Based on Remote Sensing Images. Oceanologia Etlimnologia Sinica, 50, 958-970. (In Chinese)

[2] Osborne, A.R. and Burch, T.L. (1980) Internal Solitons in the Andaman Sea. Science, 208, 451-460. https://doi.org/10.1126/science.208.4443.451

[3] Grimshaw, R., Pelinovsky, E. and Poloukhina, O. (2002) Higher-Order Korteweg-de Vries Models for Internal Solitary Waves in a Stratified Shear Flow with a Free Surface. Nonlinear Processes in Geophysics, 9, 221-235. https://doi.org/10.5194/npg-9-221-2002

[4] Ye, C.S. and Wang, M.M. (2010) Numerical Calculation on Horizontal Wave Number of Internal Waves. Henan Science, 28, 275-280. (In Chinese)

[5] Dong, C.M., Yu, K., Liu, Y., Wang, J. and Dong, Q.H. (2019) Introduction to Physical Oceanography. Science Press, Beijing. (In Chinese)

[6] Ma, R.L. (2012) Study on the Propagation Model for the Deep-Sea Internal Waves and Its Numerical Simulation. Ocean University of China, Qingdao. (In Chinese)

[7] Huang, X.D. (2013) Study on the Spatial Distributions and Temporal Variations of Internal Solitary Waves in the South China Sea. Ocean University of China, Qingdao. (In Chinese)

[8] Fliegel, M. and Hunkins, K. (1975) Internal Wave Dispersion Calculated Using the Thomson-Haskell Method. Journal of Physical Oceanography, 5, 541-548. https://doi.org/10.1175/1520-0485(1975)005<0541:IWDCUT>2.0.CO;2

[9] Ye, A.L. and Li, F.Q. (1990) Physical Oceanography. Qingdao Ocean University Press, Qingdao. (In Chinese)

[10] Liu, S.S., Liu, S.D. and Tan, B.K. (1996) Nonlinear Atmospheric Dynamics. National Defense Industry Press, Beijing. (In Chinese)

[11] Cai, S.Q. and Gan, Z.J. (1995) A Numerical Method of Internal Waves Dispersion Relation. Tropical Ocean, 14, 23-29. (In Chinese)

[12] Yang, H.L., Yang, L.G., Song, J.B., et al. (2008) Strongly Nonlinear Waves in Uniform Basic Flows and Their Dispersion Relations. Journal of Inner Mongolia University, 39, 289-293. (In Chinese)

[13] Fang, X.H., Jiang, M.S. and Du, T. (2000) Dispersion Relation of Internal Waves in the Western Equatorial Pacific Ocean. Acta Oceanologica Sinica, 19, 37-45.

[14] Munk, W.H. (1980) Internal Waves Spectra at the Buoyant and Inertial Frequencies. Journal of Physical Oceanography, 10, 1718-1728. https://doi.org/10.1175/1520-0485(1980)010<1718:IWSATB >2.0.CO;2

[15] Wang, D.Z. and Shang, E.C. (1981) Hydroacoustics. Science Press, Beijing. (In Chinese)

[16] Zhang, K.B. and Gao, H.S. (1997) A Numerical Method for Vertical Structure of Internal Waves in Ocean. Tropic Oceanology, 16, 62-67. (In Chinese) 
[17] Li, H.Y. and Du, T. (2005) Dispersion Relation of Internal Waves of the Two Layers Model K-dV Function. Journal of Hydrodynamics, Ser. A, 20, 673-679. (In Chinese)

[18] Tsien, H.S. (1955) The Poincaré-Lighthill-Kuo Method. Advances in Applied Mechanics, Vol. 4, Academic Press, New York, 281-349.

https://doi.org/10.1016/S0065-2156(08)70375-2

[19] Sakai, T. and Redekopp, L.G. (2007) Models for Strongly-Nonlinear Evolution of Long Internal Waves in a Two-Layer Stratification. Nonlinear Processes in Geophysics, 14, 31-47. https://doi.org/10.5194/npg-14-31-2007

[20] Grimshaw, R. (2002) Internal Solitary Waves. Chap. 1, Kluwer Academic, Boston, 1-27. https://doi.org/10.1007/0-306-48024-7_1

[21] Helfrich, K. and Melville, W. (2006) Long Nonlinear Internal Waves. Annual Review of Fluid Mechanics, 38, 395-425.

https://doi.org/10.1146/annurev.fluid.38.050304.092129

[22] Holloway, P. and Pelinovsky, E. (2002) Internal Solitary Waves. Chap. 2, Kluwer Academic, Boston, 29-60.

[23] Liu, A.K. and Chang, Y.S. (1998) Evolution of Nonlinear Internal Waves in the East and South China Seas. Journal of Geophysical Research, 103, 7995-8008.

https://doi.org/10.1029/97JC01918 